\title{
UNSTEADY MHD POISEUILLE FLOW THROUGH A POROUS CHANNEL UNDER AN OSCILLATING PRESSURE GRADIENT AND UNIFORM SUCTION/INJECTION
}

\author{
Judith N. Balkissoon ${ }^{1 *}$, Sreedhara Rao Gunakala ${ }^{2}$ and Victor M. Job ${ }^{3}$ \\ ${ }^{1,2,3}$ Faculty of Science and Technology, The University of the West Indies, Trinidad \\ ${ }^{1}$ Email: judith.balkissoon@my.uwi.edu *(Corresponding author) \\ ${ }^{2}$ Email: Sreedhara.Rao@sta.uwi.edu \\ ${ }^{3}$ Email: victor.job@sta.uwi.edu
}

\begin{abstract}
The unsteady magnetohydrodynamic Poiseuille flow of a viscous, incompressible, hydromagnetic fluid through a porous channel, bounded by two horizontal plates under an oscillating pressure gradient, is studied. The Brinkman's equation is used to model the flow and the Galerkin Finite Element Method is used to solve the governing equations. The values of Hartmann number $(\mathrm{Ha})$, frequency of oscillation $(\omega)$, suction/injection parameter $(S)$, the permeability parameter $(\alpha)$, time $(t)$ and the amplitude of the pressure gradient $(A)$ are discussed. The results obtained are in good agreement with the existing literature.
\end{abstract}

Keywords: MHD Poiseuille Flow; Suction/Injection; Oscillatory flow; Brinkman Equation; Finite Element Analysis

https://doi.org/10.47412/XEWX3906

\section{Introduction}

Hydromagnetics/magnetohydrodynamics (MHD), according to Davidson [1], is the study of the interaction of the flow of electrically conducting fluids, with magnetic fields. Some examples of this type of fluid are plasmas (hot ionised gases), strong electrolytes and liquid metals. In recent times MHD has been used for power generation and to stir, levitate and control flows of liquid metals during the extraction, development and production process. Important research has been done on MHD in the field of nuclear fusion as well as to develop technology to improve electric conversion efficiency, increase reliability of machinery by eliminating moving parts, and reduce emissions from coal and gas plants ([2], [1]). According to Verma and Gupta [3] there are many applications of MHD, such as, in MHD generators, air filters, water filters, water coolers, blood flow in arteries and in petroleum and chemical engineering. Studies in MHD has also helped in the understanding of space plasmas on Earth and planetary magnetospheres, the physics of the Sun, solar wind and stellar atmospheres. Petrović et al. [4] studied the effects that Hartmann number, Reynolds magnetic number, suction parameter and the porosity parameter have on flow and heat transfer characteristics. In this study, a magnetic field was applied perpendicular to the parallel plates and due to the fluid motion, a magnetic field was induced along the fluid flow direction. Among other things, they found that the velocity of flow and the induced magnetic field are reduced when the porosity parameter, Reynolds magnetic number and the Hartmann number are increased.

Poiseuille flow can be defined as fully developed, laminar flow between two parallel stationary plates, separated by some distance, driven by an applied pressure gradient [5]. Pressure gradient, according to the 
"Merriam-Webster Dictionary" [6] is 'the space rate of variation of pressure in a given direction', i.e. it is a term used to describe a difference in pressure between two points in a given direction. Hafeez and Ndikilar [7] studied the Poiseuille flow of an incompressible viscous fluid between two parallel porous plates driven by a pressure gradient $\left(\frac{d p}{d x}\right)$, with injection at the bottom and suction at the top. They found that fluid velocity is lower at the plate with injection and higher at the plate with suction. They also found that as the Reynolds number increases, the velocity decreases, and when the Reynolds number is equal to zero, the velocity profile is symmetric. In a study of oscillatory flow of hydromagnetic fluid through a porous channel filled with porous media done by Falade et al. [8], one conclusion made was that an increase in the suction/injection parameter improves the fluid temperature distribution and the flow velocity at the wall with fluid slippage. Attia and Abdeen [9] studied the unsteady magnetohydrodynamic flow and heat transfer of an incompressible, viscous, electrically conducting fluid between parallel non-conducting porous plates. The flow was driven by an exponentially decaying pressure gradient and a uniform magnetic field, as well as, uniform suction and injection were applied perpendicular to the plates. One of the conclusions they made was that the magnetic field has an influence on velocity, since as the Hartmann number increased, the velocity decreased.

Uniform suction and injection at the walls of a channel has many applications, including in magnetohydrodynamic generators, air filters, water filters, water coolers, artery blood flow and petroleum and chemical engineering [3]. Ahmed et. al [10] studied the effect of fluid suction on an oscillatory magnetohydrodynamic flow through a channel with heat transfer. They found that an increase in the magnetic field strength speeds up flow and increases the temperature, the rate of heat transfer and the amount of skin friction at the walls. Also, an increase in suction reduces flow and causes an increase in skin friction at the upper wall and a decrease in the lower. It was also noted that the fluid suction and magnetic field had a damping effect on the velocity and temperature in the core region of flow, which implies that the magnetic field and fluid suction can control the core velocity. This is particularly important in the flow of liquid metals through rectangular channels in fusion power reactors and flow of coolant fluids through channels in micro fluid devices. According to Jha and Aina [7], suction increases the heat transfer and the skin friction coefficient, while injection decreases the same. In their study on the role of suction/injection on steady, fully developed mixed convection flow in a vertical microchannel with parallel plates, it was concluded, that as suction/injection increases, the volume flow rate increases, while the rate of heat transfer decreases.

The finite element method is a very useful and effective numerical method used to solve partial differential equations which are usually based on real world problems. This method has been applied in the area of heat transfer, fluid flow, mass transport, boundary value problems and many other complex problems in the field of physics and geometry. Kala [11] used an explicit finite difference method to do a numerical study of how an unsteady magnetohydrodynamic fluid flow between two horizontal plate boundaries in a non-Darcy porous medium is affected by the magnetic field, porosity, Forchheimer, suction and pressure gradient parameters. Among other things, Kala found that fluid velocity decreases as the magnetic parameter, porosity parameter, Reynolds number or pressure gradient increase. However, the velocity increases when the suction parameter increases. Hanvey, Khare and Paul [12] used the separation of variable technique to conduct a study on unsteady MHD flow of incompressible fluid with constant pressure gradient through parallel porous plates, in an inclined magnetic field, with a transfer of heat and mass. It was concluded, that the velocity of a non-Newtonian fluid placed in an inclined magnetic field decreases as the magnetic field increases. Shit and Roy [13] used a perturbation method to do a theoretical study of pulsatile blood flow through a constricted porous channel in the presence of an external magnetic field, using the incompressible Newtonian fluid model. They found that increasing the magnetic field caused a reduction in blood flow and increasing the Reynolds number and the porosity parameter caused an increase in flow velocity. They concluded that blood flow can be controlled by applying a strong enough magnetic field. Seth, Ansari and Nandkeolyar [14] used the Laplace transform technique to investigate time-dependent hydromagnetic 
Couette flow through a channel made up of porous medium, bounded by parallel sheets with constant suction/injection at the plates and a magnetic field applied perpendicular to the direction of flow. Among other things, they concluded that the fluid flow increases when either the magnetic field, time or injection increase and decreases when suction increases.

The Brinkman equation is a combination of the Navier-Stokes equations and the Darcy Equation. It shows the transformation from slow flow governed by Darcy's law, to viscous free flow governed by NavierStokes equations [15]. Almalki et. Al [16] determined that there have been increasing interest in Brinkman's equations to model flow in porous media. Almalki and Hamden [17] investigated Brinkman's effective viscosity of fluid in a porous medium to see how it is affected by a change in the fluid viscosity, with respect to the base fluid viscosity under Poiseuille flow. A threshold value corresponding to the value of porosity higher than $98 \%$ was determined for the conversion from Darcy to Brinkman flow regime. This confirmed the possibility that Brinkman's equation is effective for high values of porosity. Verma and Gupta [3] used the Brinkman equation to model a steady magnetohydrodynamic flow in a horizontal porous channel with constant suction/injection at the walls for both Poiseuille flow and Couette-Poiseuille flow. A constant magnetic field was applied in the direction of the y-axis and the induced magnetic field was ignored. Some observations made from this study, were that the Hartmann number and the permeability and suction/injection parameter both have a strong influence on the characteristics of flow. Their studies concluded that since injection decreases skin friction, it increases flow, whereas suction slows down flow.

The above studies show examples of investigations done on Poiseuille flow, Poiseuille-Couette flow, MHD flow through porous medium, flow involving suction/injection, pressure gradient and Brinkman equations. In this paper the main focus is to investigate MHD Poiseuille flow through porous medium with an oscillating pressure gradient applied in the direction of flow. A magnetic field is also applied in the positive $\mathrm{y}$-direction and suction/injection are applied perpendicular to the horizontal plates. The effect of Hartmann number $(\boldsymbol{H a})$, frequency of oscillation $(\boldsymbol{\omega})$, suction/injection parameter $(\boldsymbol{S})$, the permeability parameter $(\boldsymbol{\alpha})$, time $(\boldsymbol{t})$ and the amplitude of the pressure gradient $(\boldsymbol{A})$ on velocity is investigated and discussed with the aid of graphs.

\section{Description of Problem}

Consider the unsteady, laminar movement of a viscous, conducting, hydromagnetic, incompressible fluid in a porous medium with constant width between two "infinitely long" horizontal parallel plates. The cartesian coordinate system chosen is in terms of $x$ and $y$, where the $x$-axis is along the centre of the medium. The width of the channel is assumed to be $2 h$. The upper and lower plates are stationary, with the lower plate positioned at $y=-h$ and the upper plate at $y=h$, as shown in Fig. 1. The fluid flow is influenced by uniform injection through the lower plate and uniform suction through the upper plate. An oscillating pressure gradient is applied in the $x$-direction and a constant magnetic field $B_{0}$ is applied perpendicular to the plates in the positive $y$-direction. It is supposed that the fluid operates like a homogeneous Newtonian fluid with unchanging density $(\rho)$, viscosity $(\mu)$ and electrical conductivity $(\sigma)$.

According to the schematic shown in (Fig. 1) and the physical description, the following assumptions are made:

1. The fluid is incompressible, i.e. $\rho$ is constant.

2. The flow is laminar and uniform.

3. The fluid has magnetic Reynolds number much smaller than 1 .

4. The pressure gradient is oscillatory.

5. The channel is permeable with permeability $K$.

6. The porous medium is described using Brinkman's model.

7. The effective viscosity is assumed to be equal to the dynamic viscosity of the fluid i.e. $\mu_{e f f}=\mu$. 


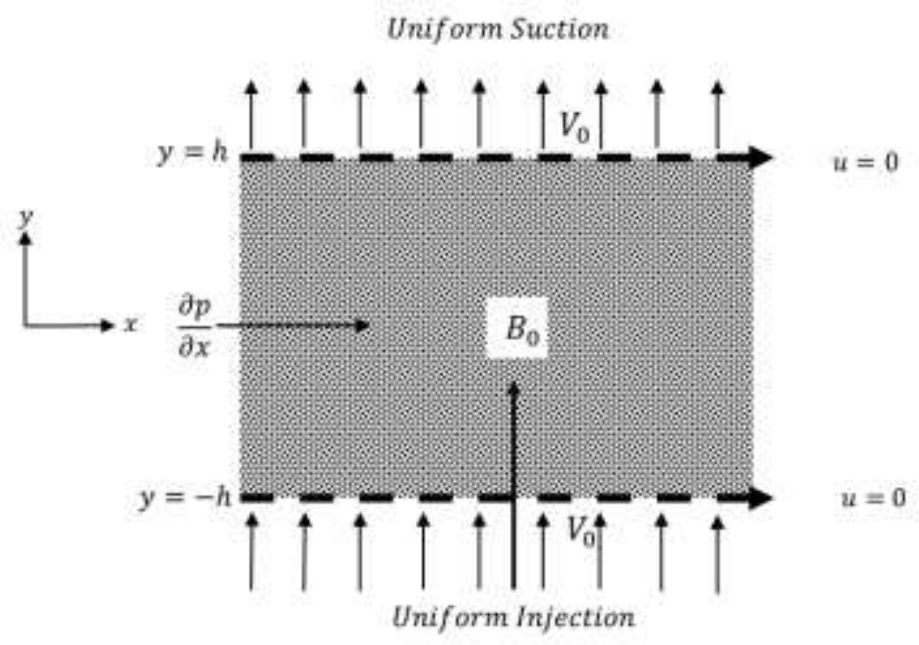

Figure 2: Schematic Diagram of Physical System

From the above assumptions, the fluid flow is governed by the Brinkman equation:

$$
\rho\left(\frac{\partial u}{\partial t}+V_{0} \frac{\partial u}{\partial y}\right)=-\frac{\partial P}{\partial x}+\mu \frac{\partial u}{\partial y^{2}}-\sigma B_{0}^{2} u-\frac{\mu}{K} u
$$

where $\rho, \sigma$ and $\mu$ are respectively, the density, electrical conductivity and coefficient of viscosity of the fluid.

The initial condition and boundary conditions are

$$
\begin{aligned}
& u=0 \text { at } t=0 \\
& u=0 \text { at } y= \pm h \text { for } t>0
\end{aligned}
$$

The problem described by equations (1) to (3) is simplified by using non-dimensional variables. The characteristic length is taken to be $h$, the characteristic velocity is used as $\frac{\mu}{\rho h}$ and the characteristic time is used as $\frac{\rho h^{2}}{\mu^{2}}$. The non-dimensional quantities are defined as follows:

$$
x^{*}=\frac{x}{h}, y^{*}=\frac{y}{h}, u^{*}=\frac{\rho h u}{\mu}, P^{*}=\frac{P \rho h^{2}}{\mu^{2}}, t^{*}=\frac{t \mu}{\rho h^{2}}
$$

Where $\alpha=\frac{h}{\sqrt{K}}$ is the permeability parameter, $S=\frac{\rho h V_{0}}{\mu}$ is the suction/injection parameter and

$H a=\sqrt{\frac{\sigma B_{0}^{2} h^{2}}{\mu}}$ is the Hartmann number. On dropping the $*$ for convenience, rearranging and using the pressure gradient as:

$$
\frac{\partial P}{\partial x}=-A(1-\cos \omega t)
$$

where $A$ is the amplitude of the pressure gradient and $\omega$ is the frequency of oscillation, equation (1) is written as:

$$
\frac{\partial u}{\partial t}+S \frac{\partial u}{\partial y}=\frac{\partial^{2} u}{\partial y^{2}}-\left(H a^{2}+\alpha^{2}\right) u+A[1-\cos (\omega t)]
$$


The non-dimensional initial and boundary conditions become:

$$
u=0 \text { at } t=0 \text { and } u=0 \text { at } y= \pm 1 \text { at } t>0
$$

The time-dependent volumetric flow rate (dimensionless) is given by [22]:

$$
Q(t)=\int_{-1}^{1} u(y, t) d y
$$

\section{Numerical Solution of the Problem}

The Galerkin Finite Element method is used to solve equation (6) numerically under the initial and boundary conditions given by equation (7). The velocity distributions are determined for different values of the parameters $S, H a, \alpha, \omega, t$ and $A$. The spatial semi-discretization was done using the weighted integral method and the Crank-Nicolson scheme is used for the time discretization. The elements are assembled and the computer software MATLAB is used to solve the system of equations.

\section{Discussion of Results}

The Galerkin finite element method was used to determine the effect of the suction/injection parameter $(S)$, Hartmann number $(\mathrm{Ha})$, permeability parameter $(\alpha)$, frequency of oscillation $(\omega)$, time $(t)$ and the amplitude of the pressure gradient $(A)$ on velocity distributions. This method was implemented using 100 quadratic elements and 100 time steps. The approximate solutions were obtained using MATLAB and were displayed in Figs. 2 to 9.

Fig. 2 shows the profiles of velocity $(u)$ for Poiseuille flow in a porous channel for different values of the permeability parameter, $\alpha=0,1,2,3$ with suction parameter, $S=1$, frequency of oscillation, $\omega=1$ and Hartmann number, $\mathrm{Ha}=2$. It can be seen that velocity increases when $\alpha$ decreases. This occurs because a decrease in the permeability parameter $\left(\alpha=\frac{h^{2}}{K}\right)$ means that there is an increase in the permeability of the porous material $(K)$ and therefore velocity increases. This is in good agreement with the study done by Verma and Gupta [3] on magnetohydrodynamic flow in a channel which is porous with constant injection and suction at the boundaries. Fig. 3 shows how the profile of velocity $u$ is affected by different values of the suction/injection parameter, using $S=0,1,2,3, H a=1, \alpha=0.00001$ and $\omega=1$. It can be observed that the graphs are non-symmetrical for $S \neq 0$ and are skewed in the direction of $y=1$, which represents the top plate where there is suction. It can also be seen that velocity $(u)$ increases as suction/injection parameter decreases. Here, the value of the permeability parameter $(\alpha)$ used is very low, which indicates that the permeability of the porous material $(K)$ was high and therefore there is an increase in velocity. These results agree with similar work done by Verma and Gupta [3] in their study of MHD flow through a porous channel with constant injection/suction.

Fig. 4 shows the profiles of velocity for Poiseuille flow for varying values of Hartmann number, $H a=$ $0,1,2,3$, with $\omega=1, S=1$ and $\alpha=1$. Fig. 5 shows how the time variation of velocity is affected by different values of Hartmann number $(\mathrm{Ha})$ using the same values of $\mathrm{Ha}, \omega, \mathrm{S}$ and $\alpha$ as stated above. Both graphs show, that as Hartmann number $(\mathrm{Ha})$ decreases, the velocity, $u$ increases. This occurs because a decrease in the Hartmann number means that the Lorentz force acting in the direction opposite to the flow decreases. So as the Hartmann number decreases, the magnetic damping force decreases and therefore the velocity $u$ increases. This result is in good accord with work carried out by Moniem and Hassanin [18], Verma and Gupta [3] and Gupta and Jain [19]. This result is also in agreement with work done by 
Manyonge, Kiema and Iyaya [20] in their study of steady magnetohydrodynamic Poiseuille flow between two infinitely long parallel porous plates in a transverse magnetic field.

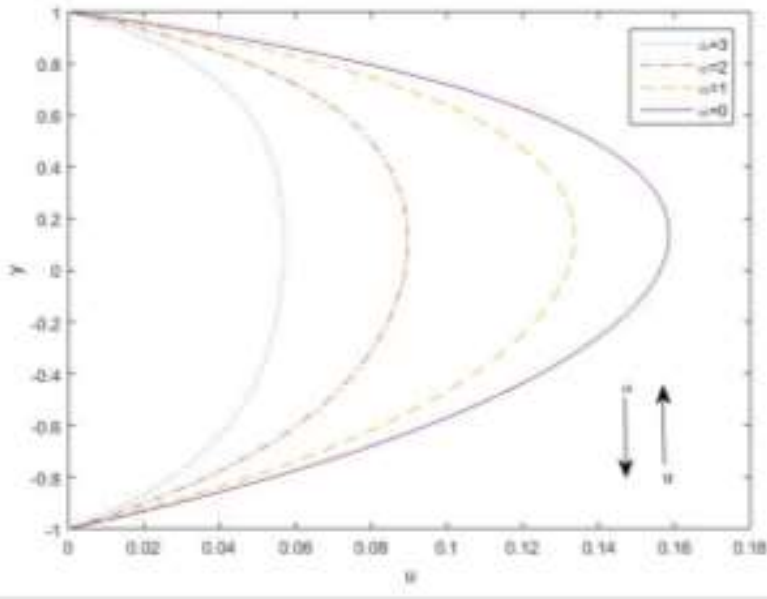

Figure 2: Velocity profile for Poiseuille flow for different values of $\alpha$.

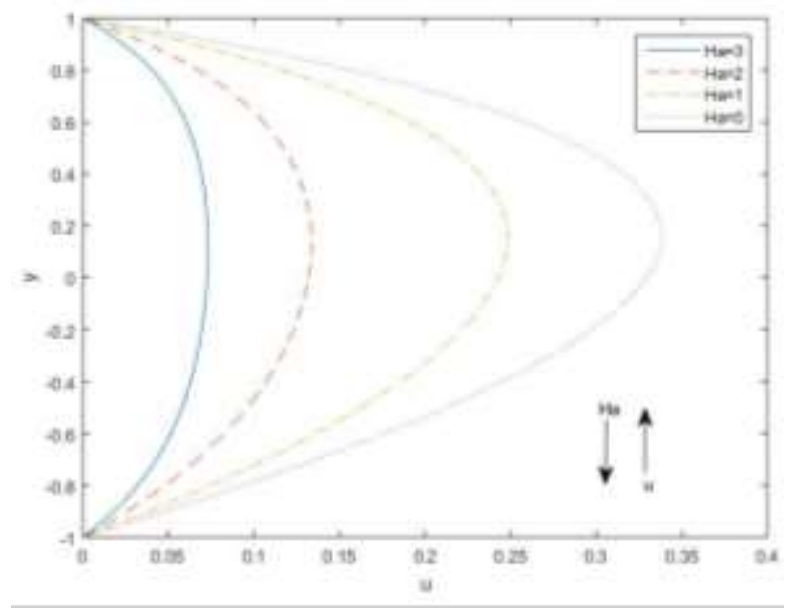

Figure 4: Velocity profiles for Poiseuille flow for different values of $\mathrm{Ha}$.

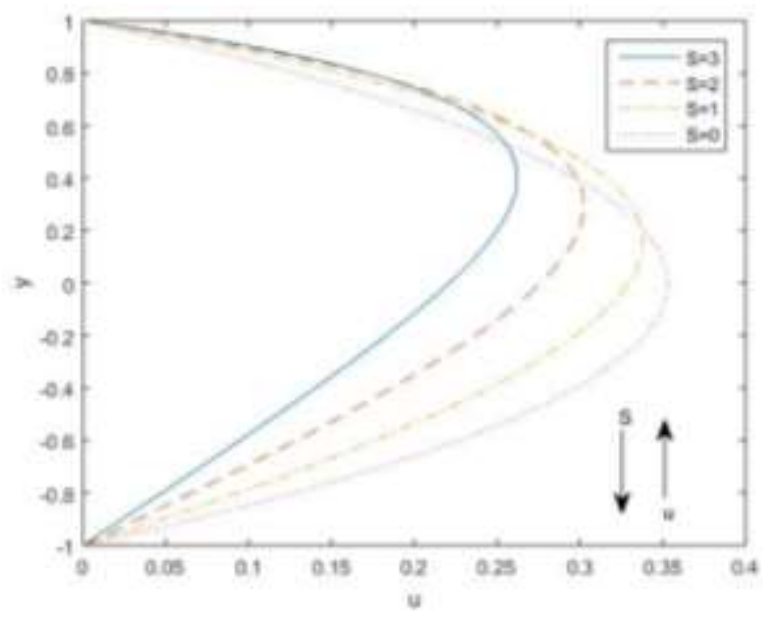

Figure 3: Velocity profile for Poiseuille flow for different values of $S$ and $\alpha=0.00001$.

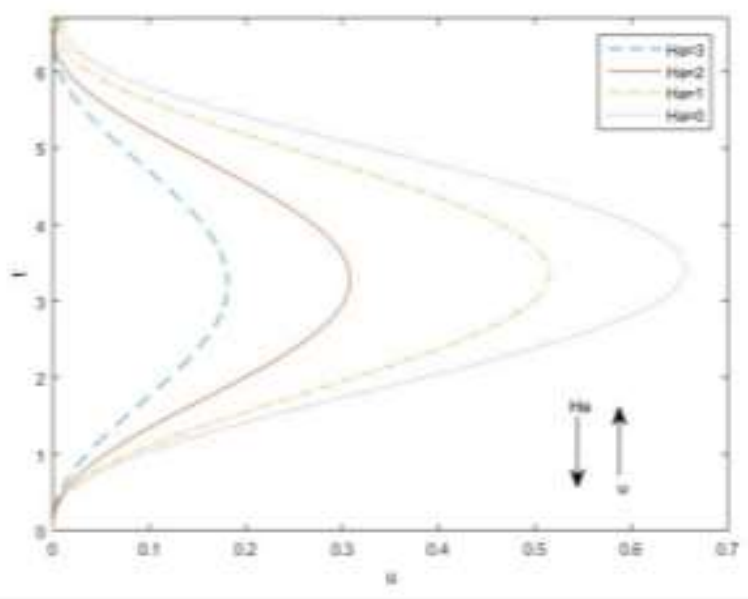

Figure 5: Effect of $\mathrm{Ha}$ number on time variation of $u$ at $y=0$.

Fig. 7 shows the profiles of velocity for Poiseuille flow for different values of the frequency of oscillation, $\omega=1,0.75,0.5$ with $H a=1, \alpha=1, A=1$ and $S=1$. From this graph it is observed that as the frequency of oscillation $(\omega)$ decreases, the velocity increases. Therefore, it can be inferred that due to the pressure gradient being $\frac{\partial P}{\partial x}=-A(1-\operatorname{Cos} \omega t)$, the pressure is increasing as the frequency of oscillations is decreasing and hence there is an increase in velocity with this increase in pressure [21]. Fig. 7 shows the effect of $\omega=1,0.75,0.5,0.25,0.1$ on time variation of $u$ with $H a=1, A=1, \alpha=1$ and $S=1$. It shows that for each value of time $t$, as the frequency of oscillation $\omega$ increases, the velocity also increases; that is, at a higher frequency $\omega$, the fluid flow is faster. 


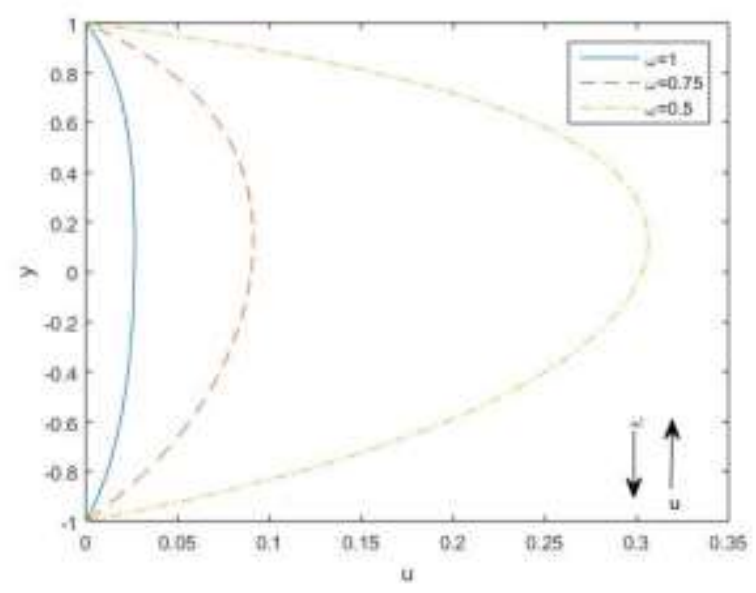

Figure 6: Velocity profiles for Poiseuille flow for different values of $\omega$.

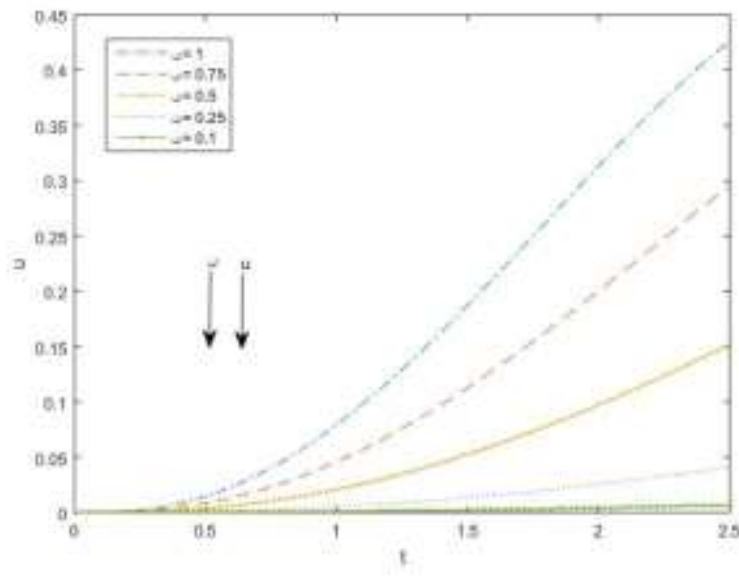

Figure 7: Effect of $\omega$ on time variation of $u$ at $y=0$.

Fig. 8 shows the effect of varying amplitude of the pressure gradient on time variation of $u$ with $A=$ $1,2,3,5,7,8, S=1, H a=1, \alpha=1$ and $\omega=1$. The graph shows that as the amplitude increases, so does the velocity $(u)$. This occurs because the value of the oscillating pressure gradient used is $\frac{\partial P}{\partial x}=$ $-A(1-\operatorname{Cos} \omega t)$. Therefore the pressure increases as the amplitude $(A)$ increases, and hence there is an increase in velocity $(u)$ [21]. Fig. 9 also shows how the time variation of $u$ is affected by the same values of the amplitude of pressure gradient used in Fig. 8 but with the frequency of oscillation, $\omega=3$. Again, $u$ increases as $A$ increases, however due to the larger value of $\omega$ used, the fluid reaches a maximum velocity in a shorter time. This may again be attributed to the value of the pressure gradient used.

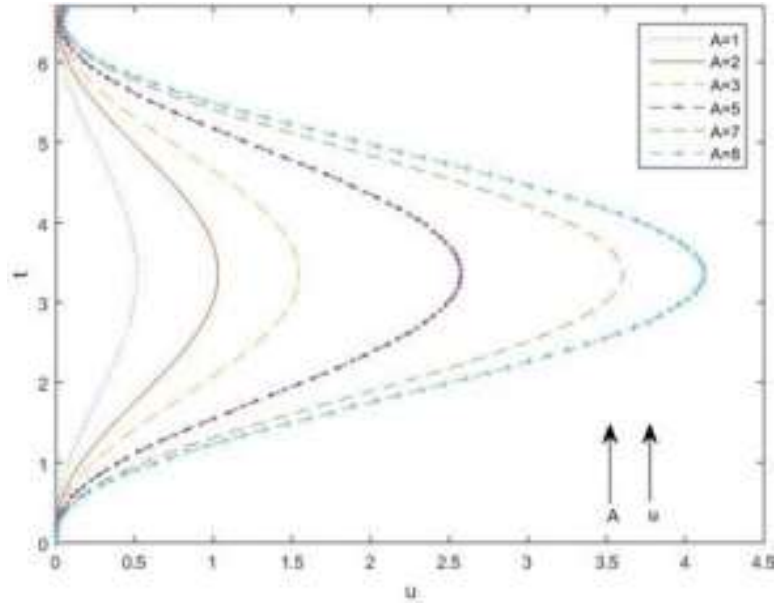

Figure 8: Effect of $A$ on time variation of $u$ at $y=0$.

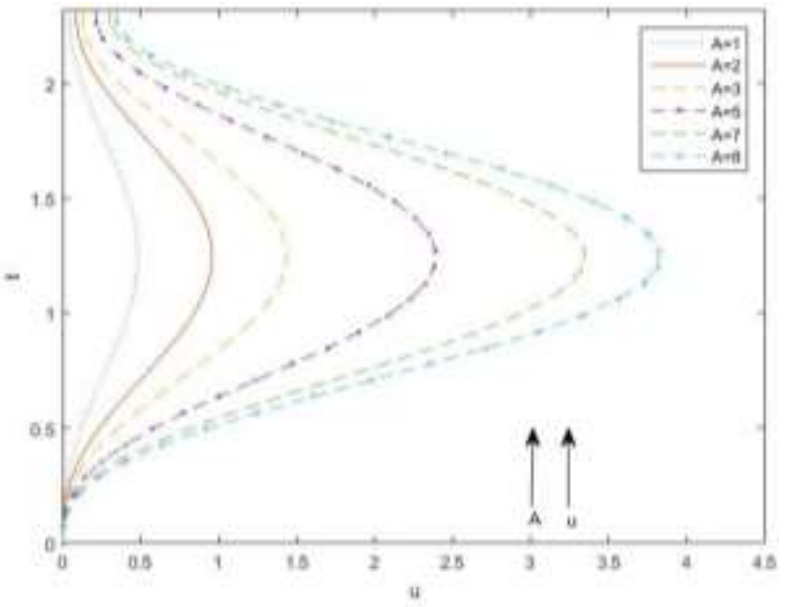

Figure 9: Velocity profile for Poiseuille flow for different values of $t$. 
Figs. 10 to 13 show the effect of the permeability parameter $(\alpha)$, the suction/injection parameter $(S)$, Hartmann number $(\mathrm{Ha})$ and the frequency of oscillation $(\omega)$ on the time-dependent volumetric flow rate $Q$. From Fig. 10 to 13 it is observed that as $\alpha, S$ and $H a$ decrease, the volumetric rate of flow $Q$ increases, however, as $\omega$ increases, $Q$ also increases. A study done by Shit and Roy [13] found that when an external magnetic field is applied to a system, the volumetric flow rate decreases.

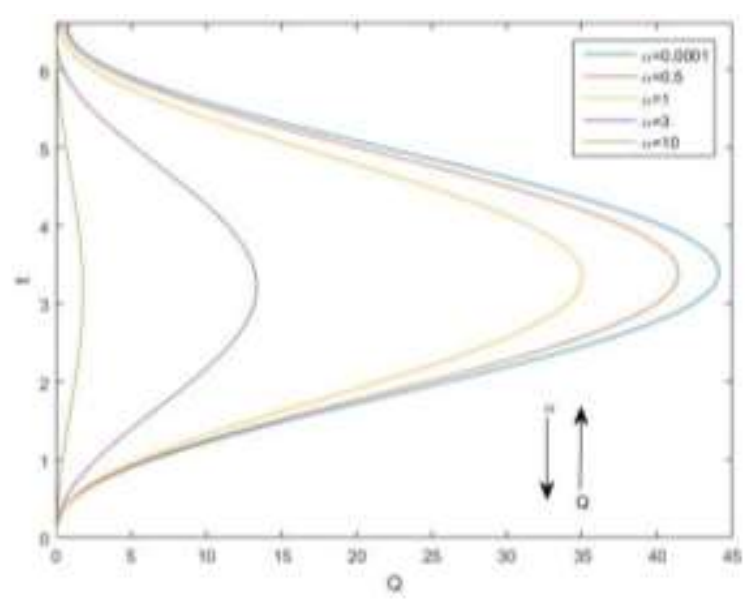

Figure 10: Effect of $\alpha$ on time variation of $Q$.

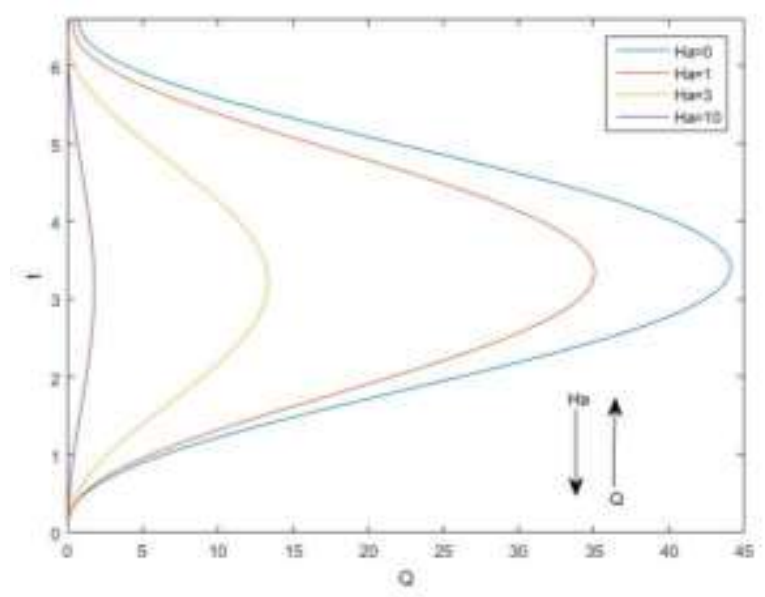

Figure 12: Effect of $H a$ on time variation of $Q$.

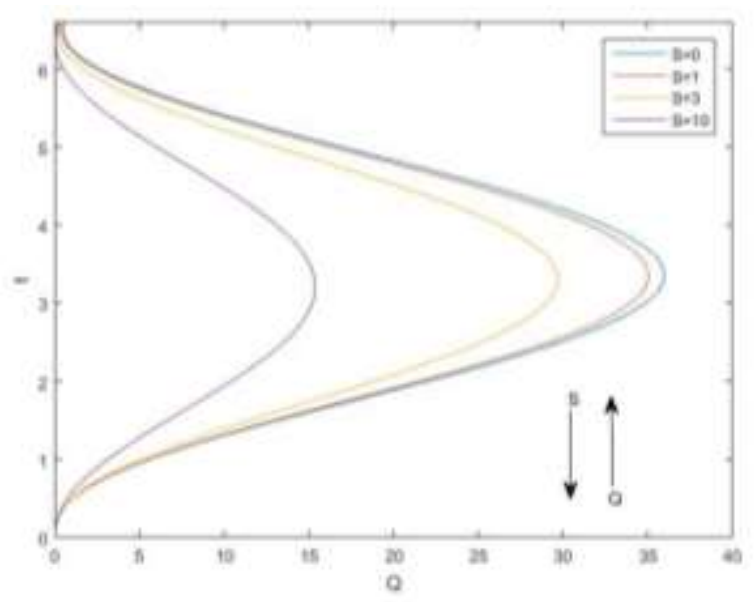

Figure 11: Effect of $S$ on time variation of $Q$.

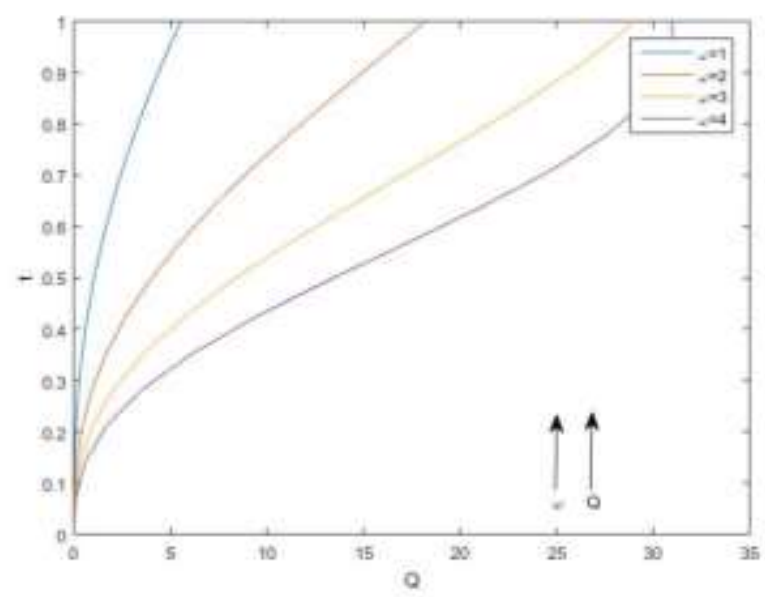

Figure 13: Effect of $\omega$ on time variation of $Q$.

\section{Conclusion}

In this study, the unsteady MHD Poiseuille flow through a porous channel under an oscillating pressure gradient and uniform suction and injection was investigated. The parameters which were varied to show the behaviour of this type of flow were the suction/injection parameter, Hartmann number, permeability parameter, frequency of oscillation, amplitude of the pressure gradient and time. The effect of the different parameters on the volumetric flow rate when varied with time was also investigated. Finite element analysis 
was used to obtain solutions to the problem and MATLAB was used to solve the finite element equations for this type of flow. It was found that velocity increases when the permeability parameter, suction/injection parameter, Hartmann number and frequency of oscillation decrease. The velocity was also observed to increase when the amplitude of the pressure gradient and time were increased. The volumetric flow rate $(Q)$ was observed to increase as the permeability parameter, suction/injection parameter and Hartmann number decreased but it increased as the frequency of oscillation increased.

\title{
Nomenclature
}

\author{
A Amplitude of the pressure gradient \\ $B_{0} \quad$ Magnetic field strength $(T)$ \\ $h \quad$ Distance between the plates and centre of the channel \\ $\mathrm{Ha}$ Hartmann number \\ $K \quad$ Permeability $\left(m^{2}\right)$ \\ $P \quad$ Pressure $(\mathrm{Pa})$ \\ Q Dimensionless volumetric flow rate \\ $\mathrm{S} \quad$ Suction/injection parameter \\ $t \quad$ Time (s) \\ $u \quad$ Horizontal flow velocity $\left(m s^{-1}\right)$ \\ $V_{0} \quad$ Suction/injection velocity $\left(m s^{-1}\right)$ \\ $x \quad$ Horizontal coordinate $(\mathrm{m})$ \\ $y \quad$ Vertical coordinate $(\mathrm{m})$

\section{Greek Letters} \\ $\alpha \quad$ Permeability parameter \\ $\mu \quad$ Viscosity (Pa s) \\ $\rho \quad$ Density $\left(\mathrm{kg} \mathrm{m}^{-3}\right)$ \\ $\sigma \quad$ Electrical conductivity $\left(\Omega^{-1} m^{-1}\right)$ \\ $\omega \quad$ Oscillation frequency of the pressure gradient $\left(s^{-1}\right)$
}

\section{References}

[1] P. A. Davidson, 2001. An Introduction to Magnetohydrodynamics. New York: Cambridge University Press.

[2] M. S. Tillack MS, N. B. Morley, 1998. Magnetohydrodynamics. In Standard Handbook for Electrical Engineers.

[3] V. K. Verma, A. K. Gupta. MHD flow in a porous channel with constant suction/injection at the walls. International Journal of Pure and Applied Mathematics 118 no. 1, (2018) 111-123.

[4] J. Petrović, Ž. Stamenković, M. Kocić, M. Nikodijević, J. Bogdanović-Jovanović. MHD flow and heat transfer in porous medium with induced magnetic field effects. Annals of the Faculty of Engineering Hunedoara 16 no. 1, (2018) 171-174.

[5] Y. A. Cengel, J. M. Cimbala. 2006. Fluid mechanics: fundamentals and applications. New York: McGraw-Hill Education.

[6] Merriam Webster Inc. 1994. Merriam-Webster's Dictionary of English Usage. Massachusetts: Merriam-Webster, Incorporated.

[7] H. Y. Hafeez, C. E. Ndikilar. Flow of viscous fluid between two parallel porous plates with bottom injection and top suction. Progress in Physics 10 no. 1, (2014) 49-51. 
[8] J. A. Falade, J. C. Ukaegbu, A. C. Egere, S. O. Adesanya. MHD oscillatory flow through a porous channel saturated with porous medium. Alexandria Engineering Journal, 56 no. 1, (2017) 147-152.

[9] H. A. Attia, M. A. M. Abdeen. Unsteady hartmann flow with heat transfer of a viscoelastic fluid under exponential decaying pressure gradient. Engineering Mechanics 19 no. 5, (2012) 37-44.

[10] N. Ahmed, A. H. Sheikh, D. P. Barua. Effect of fluid suction on an oscillatory MHD channel flow with heat transfer. Applications \& Applied Mathematics 11 no. 1, (2016) 266-284.

[11] B. S. Kala. Numerical study of the effects of suction and pressure gradient on an unsteady mhd fluid flow between two parallel plates in a non-darcy porous medium. Asian Research Journal of Mathematics 3 no. 4, (2017) 1-14.

[12] R. R. Hanvey, R. R. Khare, A. Paul. MHD flow of incompressible fluid through parallel plates in inclined magnetic field having porous medium with heat and mass transfer. International Journal of Scientific and Innovative Mathematical Research (IJSIMR) 5 no. 4, (2017) 18-22.

[13] G. C. Shit, M. Roy. 2012. Hydromagnetic pulsating flow of blood in a constricted porous channel: a theoretical study. In Proceedings of the World Congress on Engineering (vol. 1, pp. 83-88) WCE.

[14] G. S. Seth, M. S. Ansari, R. Nandkeolyar. Unsteady hydromagnetic couette flow within a porous channel. Tamkang Journal of Science and Engineering 14 no. 1, (2011) 7-14.

[15] M. Parvazinia, V. Nassehi, R. J. Wakeman, M. H. R. Ghoreishy. Finite element modelling of flow through a porous medium between two parallel plates using the Brinkman equation. Transport in porous media 63 no. 1, (2006) 71-90.

[16] W. S. J. Almalki, M. H. Hamdan, M. T. Kamel. 2010. Analysis of flow through layered porous media. In Proceedings of the 12th WSEAS International Conference on Mathematical Methods, Computational Techniques and Intelligent Systems (pp. 182-189) WSEAS.

[17] W. S. Almalki and M. H. Hamdan. Investigations in effective viscosity of fluid in a porous medium. International Journal of Engineering Research and Applications 6 no. 4, (2016) 41-51.

[18] A. A. Moniem, W. S. Hassanin. Solution of MHD flow past a vertical porous plate through a porous medium under oscillatory suction. Applied Mathematics 4 no. 4, (2013) 694-702.

[19] V. G. Gupta, A. Jain. An analysis of unsteady MHD Couette flow and heat transfer in a rotating horizontal channel with injection/suction. International Journal of Latest Technology in Engineering, Management \& Applied Science 5 no. 4, (2016) 2278-2540.

[20] W. A. Manyonge, D. W. Kiema, C. C. W. Iyaya. Steady MHD poiseuille flow between two infinite parallel porous plates in an inclined magnetic field. International journal of pure and applied mathematics 76 no. 5, (2012) 661-668.

[21] Y. Nakayama, R. F. Boucher. 1999. Introduction to fluid mechanics. Oxford: ButterworthHeinemann.

[22] I. Tosun. 2007. Modeling in transport phenomena: a conceptual approach. Amsterdam: Elsevier. 